\title{
Aspectos Reprodutivos das Mulheres Climatéricas do Programa de Saúde da Familia em Cuiabá
}

\author{
Reproductive Aspects of Climacteric Women of the Family Health Program in Cuiabá \\ Vivaldo Naves de Oliveira, Joaquim Gonçalves Valente, Sebastião Freitas de Medeiros
}

\begin{abstract}
RESUMO
Objetivo: caracterizar os aspectos reprodutivos das mulheres climatéricas do Programa de Saúde da Família de Cuiabá.

Métodos: o estudo foi o de corte transversal e inclui 354 mulheres com idade entre 40 e 65 anos, atendidas pelo Programa de Saúde da Família do Distrito Centro-Oeste de Cuiabá. Na coleta de dados utilizou-se um questionário previamente testado no Ambulatório de Climatério do Hospital Universitário Júlio Muller. Os dados foram analisados pelo teste $Z$ e $\chi^{2}$ para

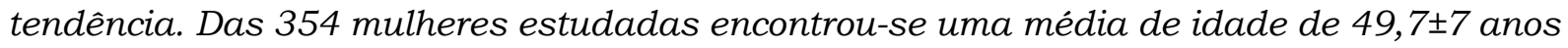
e mediana de 48 anos. Desse total, 243 (68,6\%) eram naturais de Mato Grosso, sendo as outras migrantes de vários Estados. A maioria era casada (65,8\%), branca (48,0\%), católica $(73,4 \%)$ e com baixa escolaridade: $62,4 \%$ cursaram, mas não concluíram, o primeiro grau e 19,2\% eram analfabetas. Do total, 84,3\% estavam ocupadas em atividades exclusivamente domésticas e mais da metade $(58,2 \%)$ pertencia à classe social mais baixa.

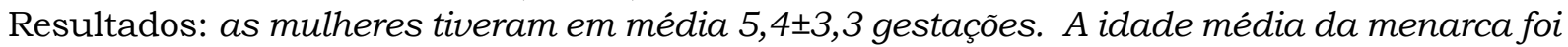

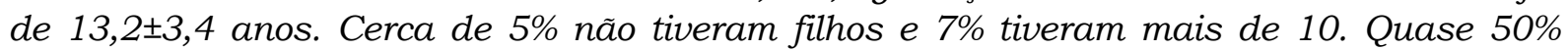
tiveram sua primeira gravidez na adolescência e $14 \%$ após os 40 anos. A menopausa foi cirúrgica em 25\% das mulheres. Cerca de 65\% delas (229) tinham sido submetidas a cirurgia ginecológica prévia: 78\% à laqueadura tubária, 20\% à histerectomia e 7\% à ooforectomia bilateral.

Conclusão: quase 65\% tinham sido submetidas a cirurgia ginecológica, sendo as cirurgias mais freqüentes a laqueadura tubária e a histerectomia total. Após a constituição da prole o método mais freqüente para planejamento familiar foi a ligadura de trompas.
\end{abstract}

PALAVRAS-CHAVE: Climatério. Laqueadura tubária. Histerectomia.

\section{Introdução}

Climatério define o período de vida e menopausa a parada das menstruações. Ainda que o climatério seja um fenômeno fisiológico, pode ter conseqüências negativas sobre vários sistemas orgânicos. Em média a menopausa natural ocorre aos 50 anos, havendo variações entre países. Há informações de que, nos países mais desenvolvi-

Ambulatório de Climatério do Hospital Universitário Júlio Muller

Departamento de Ginecologia e Obstetrícia da Faculdade de Ciências Médicas da Universidade Federal de Mato Grosso

Correspondência:

Sebastião Freitas de Medeiros

Rua Marechal Deodoro, 1055/1302 - Centro

78005-101 - Cuibá - MT

e-mail: sdemed@terra.com.br

Tel.: (65) 322-6043 ou 615-7330 dos, ocorra em torno de 51 anos e, em países emergentes, em torno de 48 anos. No Brasil, a menopausa ocorre em média aos 48,5 e em população de baixa renda de Cuiabá, aos $48 \operatorname{anos}^{1}$. Ainda que esta idade não tenha mudado no último século, alguns fatores biológicos e reprodutivos podem afetar a função ovariana, influenciando a sintomatologia e a idade de ocorrência da menopausa ${ }^{2,3}$. A saúde e a qualidade de vida da mulher climatérica podem ser asseguradas pela introdução de programas educativos acerca do estilo de vida, dieta e cuidados preventivos sistematizados. As repercussões do hipoestrogenismo sobre o sistema reprodutor são percebidas em médio prazo em quase todas as mulheres. As infecções genitais e urinárias, o sangramento, o ressecamento vaginal, ardência miccional e dispareunia são motivos freqüentes de procura médica. A influência da paridade, da manutenção da atividade sexual e das 
cirurgias sobre os genitais na saúde-qualidade de vida desta população não está mensurada. Em algumas sociedades é alta a prevalência da esterilização cirúrgica, da histerectomia e mesmo da ooforectomia profilática durante a histerectomia realizada após a menopausa.

Os estudos sobre os efeitos destes fatores sobre a menopausa sugerem retardo na sua ocorrência nas mulheres com ciclos mais longos e maior paridade ${ }^{4}$. Tem-se discutido a influência negativa da laqueadura tubária sobre a função ovariana, mas faltam estudos avaliando seus determinantes e o papel desta cirurgia na idade de ocorrência da menopausa. Em relação à histerectomia, cirurgia efetuada em larga escala em nosso meio, parece sofrer influência da classe social, paridade e padrão de sangramento. Esta cirurgia tem menor prevalência no Reino Unido mas é comum também nos Estados Unidos ${ }^{5}$. São inconsistentes os estudos que avaliaram a função ovariana após a histerectomia, havendo relatos que variam de nenhuma alteração até a ocorrência de efeitos adversos severos. As controvérsias acerca da preservação ou não dos ovários na histerectomia realizada durante o climatério devem considerar a ação preventiva sobre o câncer de ovário e as possiveis desvantagens sobre sexualidade, doenças cardiovasculares e perda da massa óssea ${ }^{6}$. Neste estudo descrevem-se os aspectos reprodutivos e a história cirúrgica ginecológica de mulheres climatéricas, menopausadas ou não, de uma comunidade de baixa renda.

\section{Pacientes e Métodos}

Este estudo de corte transversal analisa os aspectos reprodutivos em uma população de mulheres com idade entre 40 e 65 anos do Programa de Saúde da Família do Distrito Centro-Oeste de Cuiabá. Aspectos obstétricos e ginecológicos/cirúrgicos foram registrados. Em 21 domicilios, 5,9\% de um total de 1142 visitados, a entrevista não foi realizada. $O$ instrumento utilizado para a coleta de dados foi estruturado em 6 domínios, com 39 questões de fácil compreensão. Durante a entrevista as mulheres eram identificadas e perguntadas acerca dos aspectos reprodutivos e antecedentes cirúrgicos ginecológicos. A equipe de entrevistadores foi composta pelo pesquisador principal e cinco acadêmicos do curso de medicina, previamente treinados no Ambulatório de Climatério do Hospital Universitário Júlio Muller, para familiarização com a aplicação do instrumento. A obtenção do termo de consentimento livre e esclarecido, o contato preliminar e a entrevista para a coleta de dados seguiram os critérios de natureza ética preconizados pela resolução n. ${ }^{\circ}$ 196/96 do Conselho Nacional de Saúde do Ministério da Saúde. Este estudo foi aprovado pelo Comitê de Ética do Hospital Universitário Júlio Muller (HUJM). As características sociodemográficas desta população foram descritas em publicação recente $^{7}$. Em sintese, nas 354 mulheres estudadas a idade média foi de $49,7 \pm 7$ anos e a mediana de 48 anos, sendo que mais da metade das mulheres $(56,5 \%)$ tinha menos de 50 anos. Duzentos e quarenta e três (68\%) eram naturais de Mato Grosso, sendo as outras migrantes dos Estados de Minas Gerais $(6,2 \%)$, São Paulo $(6,2 \%)$, Paraná $(4,0 \%)$ e Mato Grosso do Sul (3,7\%). A maioria destas mulheres era casada $(65,8 \%)$, de cor branca $(48,0 \%)$ e de religião católica $(73,4 \%)$. Considerando a escolaridade, $62,4 \%$ tinham cursado o primeiro grau de modo incompleto e 19,2\% eram analfabetas. Cerca de $84,3 \%$ das mulheres estavam ocupadas com atividades exclusivamente domésticas, sendo que $6,8 \%$ desenvolviam atividades comerciais e $4 \%$ dedicavam-se ao ensino. Nesta população não se identificou nenhum indivíduo pertencente à classe econômica A, sendo que mais da metade $(58,2 \%)$ pertencia à classe $\mathrm{D}, 25 \%$ à classe $\mathrm{C}$ e cerca de $7 \%$ à classe $\mathrm{E}$. A descrição dos resultados foi feita por meio da distribuição das diversas variáveis em tabelas. Os coeficientes de assimetria de Pearson e de curtose foram utilizados para avaliação da normalidade das distribuições. Para a comparação entre proporções utilizou-se o teste $Z$ e $\chi^{2}$ para tendência quando necessário. Quando $\mathrm{p}<0,05$ os resultados foram considerados estatisticamente significantes.

\section{Resultados}

As idades em que ocorreram a menarca $(13,2 \pm 3,4$ anos) e a menopausa ( $48 \pm 4,2$ anos) desta população foram divulgadas recentemente ${ }^{1}$. Estas mulheres tiveram em média $5,4 \pm 3,3$ gestações, variando de nenhuma a 16 (Tabela 1). Como a distribuição mostrou assimetria positiva, a mediana foi estimada em 5 gestações. Cerca de $5 \%$ das mulheres nesta população de baixa renda nunca tiveram filho. Por outro lado, $7 \%$ tiveram mais de 10. A análise das idades em que ocorreram a primeira e a última gravidez (Tabela 2) mostrou que quase $50 \%$ das mulheres tiveram sua primeira gravidez ainda na adolescência e $80 \%$ até os 25 anos de idade. Em relação à última gravidez, 50\% delas tiveram seu último filho na $4^{\text {a }}$ década de vida e $14 \%$ ainda pariram aos 40 anos de idade, ou mesmo após. 
Tabela 1 - Distribuição das mulheres em idade climatérica do Programa de Saúde da Família do Distrito Centro-Oeste, na área de abrangência do bairro Ribeirão da Ponte, pelo número de gestações - Cuiabá-MT*.

\begin{tabular}{crr}
\hline Gestações & $\mathbf{n}$ & $\%$ \\
\hline 0 & 17 & 4,8 \\
1 & 12 & 3,4 \\
2 & 29 & 8,2 \\
3 & 60 & 16,9 \\
4 & 51 & 14,5 \\
5 & 36 & 10,2 \\
6 & 32 & 9,0 \\
7 & 29 & 8,2 \\
8 & 22 & 6,2 \\
9 & 20 & 5,6 \\
10 & 20 & 5,6 \\
11 & 6 & 1,7 \\
12 & 11 & 3,1 \\
$\geq 13$ & 9 & 2,6 \\
Total & 354 & 100,0 \\
\hline${ }^{*}$ Coeficiente &
\end{tabular}

${ }^{*}$ Coeficiente de assimetria de Pearson 0,260 e de curtose 0,245

Tabela 2 - Distribuição das mulheres em idade climatérica do Programa de Saúde da Família do Distrito Centro-Oeste, segundo as idades da primeira e última gestação Cuiabá/MT.

\begin{tabular}{crrrr}
\hline Faixa Etária & \multicolumn{2}{c}{ Primeira gravidez } & \multicolumn{2}{c}{ Última gravidez } \\
& $\mathbf{n}$ & \multicolumn{1}{c}{$\%$} & $\mathbf{n}$ & \multicolumn{1}{c}{$\%$} \\
\hline $10-14$ & 19 & 5,6 & 00 & 0,0 \\
$15-19$ & 166 & 49,3 & 04 & 1,2 \\
$20-24$ & 95 & 28,2 & 38 & 11,4 \\
$25-29$ & 36 & 10,7 & 79 & 23,6 \\
$30-34$ & 11 & 3,3 & 92 & 27,6 \\
$35-39$ & 7 & 2,0 & 75 & 22,4 \\
$40-44$ & 3 & 0,9 & 39 & 11,7 \\
$>44$ & 0 & 0,0 & 07 & 2,1 \\
Total & 337 & 100,0 & 334 & 100,0 \\
\hline 17
\end{tabular}

Em relação ao passado cirúrgico-ginecológico, incluindo todas as mulheres, independente de já terem tido ou não a menopausa, cerca de $65 \%$ (229 mulheres) referiram algum tipo de cirurgia ginecológica prévia. Entre as 229 operadas, $78 \%$ tinham sido submetidas à laqueadura tubária e 20,5\% à histerectomia, acompanhada ou não de ooforectomia (Tabela 3). A verificação de possivel associação entre histerectomia, com ou sem ooforectomia, e paridade mostrou que $8,5 \%$ das histerectomizadas eram nuliparas, $25,5 \%$ tinham entre 1 e 3 filhos e 46,8\% tinham 4 a 7 filhos; $19,2 \%$ das histerectomias foram efetuadas em mulheres com 7 a 16 filhos $\left(\chi^{2}\right.$ TR $\left.=0,71 ; p>0,05\right)$.
A laqueadura tubária foi feita em $30,7 \%$ das mulheres que tiveram entre 1 a 3 filhos, em 48,0\% daquelas com 4 a 7 filhos e em $21,3 \%$ das restantes $\left(\chi^{2}\right.$ TR $\left.=0,73 ; p>0,05\right)$. Não se detectou associação entre ooforectomia unilateral e paridade.

Tabela 3 - Distribuição das mulheres em idade climatérica do Programa de Saúde da Família do Distrito Centro Oeste, submetidas previamente à cirurgia pélvica, segundo 0 tipo de cirurgia - Cuiabá-MT.

\begin{tabular}{lcc}
\hline Tipo de cirurgia & $\mathbf{n}$ & $\%$ \\
\hline Histerectomia total & 22 & 9,9 \\
Histerectomia com ooforectomia bilateral & 15 & 6,5 \\
Histerectomia com ooforectomia unilateral & 10 & 4,4 \\
Ooforectomia unilateral & 2 & 0,9 \\
Ooforectomia bilateral & 1 & 0,4 \\
Laqueadura de trompas & 179 & 78,2 \\
Total & 229 & 100,0 \\
\hline
\end{tabular}

A análise das mulheres submetidas a histerectomia por escolaridade mostrou que $17 \%$ eram analfabetas, $57,5 \%$ cursaram o primeiro grau, $14,9 \%$ o segundo grau e apenas $10,6 \%$ tinham curso superior $\left(\chi^{2} \mathrm{TR}=2,22 ; p>0,05\right)$. Não se detectou também associação entre a laqueadura tubária e a escolaridade das mulheres (Tabela 4).

Tabela 4 - Distribuição comparativa entre as mulheres submetidas a laqueadura tubária em toda a população estudada, segundo a escolaridade*

\begin{tabular}{lrccc}
\hline Grau de & \multicolumn{2}{c}{ População total } & \multicolumn{2}{c}{$\begin{array}{c}\text { População com } \\
\text { escolaridade }\end{array}$} \\
& $\mathbf{n}$ & $\%$ & $\mathbf{n}$ & $\%$ \\
\hline Nenhuma & 68 & 19,2 & 23 & 13 \\
$1^{\circ}$ grau incompleto & 221 & 62,4 & 121 & 67,6 \\
$1^{\circ}$ grau completo & 13 & 3,7 & 08 & 4,5 \\
$2^{\circ}$ grau incompleto & 13 & 3,7 & 07 & 3,9 \\
$2^{\circ}$ grau completo & 10 & 2,8 & 03 & 1,7 \\
Superior & 29 & 8,2 & 17 & 9,5 \\
Total & 354 & 100,0 & 179 & 100,0 \\
\hline
\end{tabular}

${ }^{*} p>0,05$ para todas as comparações nos diferentes estratos e nas análises para tendência.

Considerando apenas as 184 pacientes já no período pós-menopausa, em $25 \%$ (46) a menopausa foi cirúrgica, sendo em $6(13 \%)$ antes dos 40 anos de idade, em 26 (56,5\%) entre os 40 e 49 anos e em $10(21,7 \%)$ entre os 50 e 59 anos. A ooforectomia bilateral foi realizada em 16 mulheres $(6,9 \%)$, sendo quinze durante histerectomia. Destas, $62,5 \%$ pertenciam à classe social D e E e 37,2\% 
às classes $\mathrm{B}$ e $\mathrm{C}$. Cerca de $81,2 \%$ tinham idade acima de 50 anos. A ooforectomia bilateral foi efetuada em $12,5 \%$ de nuligestas, $18,7 \%$ das mulheres com 1 a 3 filhos, 31,3\% daquelas com 4 a 7 filhos e em $37,55 \%$ das que tiveram sete ou mais filhos. O número de casos não permitiu análise da tendência da ooforectomia de ser mais prevalente com o aumento da paridade.

\section{Discussão}

A população analisada neste estudo é, na sua maioria, de mulheres climatéricas brancas, casadas, católicas, de baixa escolaridade, de baixo nivel social econômico e dedicadas às atividades domésticas. Mais de 50\% não tinham completado 50 anos e 30\% tinham entre 50 e 60 anos. Esse perfil é semelhante a populações descritas em outros estudos nacionais ${ }^{8,9}$.

$\mathrm{Na}$ análise dos aspectos reprodutivos, a paridade foi alta, cerca de 5,4 gestações por mulher. Este achado pode ser explicado pela baixa escolaridade e desinformação destas mulheres acerca dos métodos disponíveis para o planejamento familiar quando estavam na fase reprodutiva. Halbe et al. ${ }^{10}$ também encontraram média de 5,5 gestações em uma amostra de 1319 mulheres, com idade entre 41 e 62 anos, atendidas no Serviço de Ginecologia Endócrina e Climatério do Hospital das Clínicas da Faculdade de Medicina da Universidade de São Paulo. Taxa um pouco menor, de 3,9 gestações por mulher, foi descrita em 102 mulheres, com idade média de 52,6 anos, atendidas no Ambulatório de Menopausa de Departamento de Tocoginecologia do Centro de Atenção Integral à Saúde da Mulher da Universidade Estadual de Campinas (UNICAMP) ${ }^{11}$. Este decréscimo na paridade em Campinas espelha a melhor condição de vida naquela região. Multiparidade de 4 a 7 filhos ainda tem sido observada em países emergentes ou culturalmente diferentes ${ }^{9}$. A importância das diferenças socioeconômica e cultural das populações sobre a paridade é destacada pelo achado de taxas de 1 a 2 gestações por mulher nos países desenvolvidos.

Em relação à contracepção cirúrgica, a taxa de $78 \%$ de laqueadura tubária observada aqui é extremamente alta, mesmo se comparada à taxa de $40 \%$ encontrada no país ${ }^{12}$. Esta taxa tão alta tem análise complexa. É possivel que o baixo nível cultural e/ou falta de esclarecimento e disponibilidade de outros métodos tenham influenciado. Além dos fatores desfavoráveis já apontados, estímulo à laqueadura em massa tem sido atitude freqüente nos meses que precedem as eleições em
Mato Grosso nos últimos vinte anos. Não foi possível identificar associação de laqueadura tubária com escolaridade e paridade, embora esta cirurgia tenha sido $50 \%$ mais prevalente nas mulheres com paridade entre 4 e 7 filhos quando comparadas com as que tiveram menos de quatro. Este achado corrobora estudo nacional recente, executado com controle adequado, mostrando a existência de possiveis variáveis confundidoras. Embora o presente estudo não tenha feito esta verificação, há indícios de que a esterilização cirúrgica aumente a necessidade de feitura de histerectomia no futuro ${ }^{13}$.

A histerectomia, com ou sem ooforectomia, foi relatada por $13 \%$ das mulheres incluidas neste estudo e representou $20,5 \%$ das cirurgias pélvicas. As repercussões anatomofuncionais sobre os ovários, idade da menopausa e gravidade dos sintomas do climatério nas mulheres histerectomizadas persistem sem avaliação adequada. É discutivel se a histerectomia pode determinar diminuição precoce da reserva ovariana. Relata-se o aparecimento de sintomas vasomotores mais graves, e antes do esperado para a população geral, quando a histerectomia é realizada antes ou após os $40 \operatorname{anos}^{14}$. Vários fatores podem influenciar a taxa de histerectomia. Parece que, nos países desenvolvidos, $20 \%$ das mulheres serão submetidas a histerectomia antes de ocorrer a menopausa ou até a idade de 55 anos $^{15}$. Descreve-se ainda taxa de $31 \%$ aos 60 anos em Ede, Holanda, e de 34\% na Finlândia $^{16,17}$. Neste estudo, cerca de $62 \%$ das histerectomias tinham sido realizadas antes dos 55 anos, não havendo tendência associativa entre histerectomia e idade. Este achado corrobora o de Vessey et al. ${ }^{15}$ mostrando tendência de aumento nas idades entre 30-34 e 35-39 anos, permanecendo estáveis a partir desta idade.

Classe social também parece não influenciar a taxa de histerectomia ${ }^{15}$. Embora esta análise não tenha sido feita, há que se considerar que quase a totalidade das mulheres analisadas aqui pertenciam às classes sociais desfavoráveis. Em adição, não se demonstrou tendência entre escolaridade e taxa de histerectomia $\left(\chi^{2} \mathrm{TR}=3,15\right.$ e $\chi^{2}$ $\mathrm{c}=3,84 ; \mathrm{p}>0,05)$. Não se observou também influência de paridade nas taxas de histerectomia $\left(\chi^{2}\right.$ $c=3,84$ e $\chi^{2}$ TR $\left.=0,71 ; p>0,05\right)$. Este último achado é discordante daquele observado por Vessey et al. ${ }^{15}$ na Inglaterra, mostrando associação positiva modesta entre estas duas variáveis ${ }^{15}$. Informações acerca de eventual beneficio da histerectomia na diminuição do risco de câncer de mama são es$\operatorname{cassas}^{18}$, sendo que esta associação não foi examinada no presente estudo. A menor prevalência da histerectomia nesta população de baixa renda, quando comparada com outras populações, pode 
refletir baixa percepção/valorização das modificações somáticas ou resistência aos processos cirúrgicos como instrumento terapêutico.

A prevalência de $4,5 \%$ de ooforectomia bilateral nesta população de climatéricas entre 40-65 anos, cerca de $7 \%$ das cirurgias pélvicas, foi associada à histerectomia em quase $94 \%$ das vezes. Percebeu-se aqui tendência no aumento da ooforectomia com a paridade, mas o número pequeno de casos não permitiu análise estatística. Com esta mesma limitação, não se identificou também associação entre ooforectomia e a classe social. Embora o estudo não analise diretamente este aspecto, sugere que a retirada das gônadas deve ter sido profilática na quase totalidade dos procedimentos. A ooforectomia profilática durante histerectomia por doença uterina benigna tem sido debatida mas permanece conduta controvertida. Os defensores da ooforectomia profilática consideram a prevalência de câncer de ovário de um caso a cada 70 mulheres, a baixa sensibilidade/ especificidade dos métodos disponíveis para sua identificação precoce/ prevenção e o fato de que 4 a $14 \%$ das pacientes com câncer de ovário relatam histerectomia prévia ${ }^{19}$. Nos Estados Unidos, cerca da metade das histerectomias envolvem também a ooforectomia e a literatura mundial relata ooforectomia profilática na histerectomia em 50 a $66 \%$ das vezes ${ }^{20}$. Neste estudo, incluindo 47 histerectomias, os ovários foram retirados em 15 , cerca de $32 \%$ dos procedimentos.

\section{ABSTRACT}

Purpose: to evaluate the reproductive aspects in climacteric women of the Family Health Program in Cuiabá.

Methods: it was a cross-sectional study including 354 women, with ages between 40 and 65 years, attended at the Central-Western District Family Health Program of Cuiabá. A pretested questionnaire with specific questions regarding the present study was used. Data were analyzed using the $Z$ test and $\chi^{2}$ test for trend. The mean age of the 354 women was $49.7 \pm 7$ years and the median 48 years. A total of 243 (68.6\%) were born in Mato Grosso, and the others were migrants from different states. Most were married (65.8\%), white (48.0\%), catholic (73.4\%), and had low schooling: $62.4 \%$ did not finish the elementary school and $19.2 \%$ were illiterate. About $84 \%$ were occupied only in domestic activities, and more than a half (58.2\%) belonged to a lower social class.

Results: the average number of pregnancies was 5.4 \pm 3.3 . Nearly $5 \%$ were nulliparous and $7 \%$ had more than ten children. Almost $50 \%$ had the first pregnancy in adolescence and $14 \%$ after the age of 40 years. A total of 229 women (65\%) were submitted to pelvic surgery: $78 \%$ to tubal sterilization, 20\% to hysterectomy, and $7 \%$ to bilateral oophorectomy. Menopause was due to hysterectomy/ oophorectomy in $25 \%$ of the women.

Conclusion: almost $65 \%$ of the women were submitted to a pelvic surgery. The most frequent surgery was tubal sterilization followed by total hysterectomy.

KEY WORDS: Menopause.Tubal ligation. Histerectomy.

\section{Referências}

1. Oliveira VN, Fontes CJF, Medeiros SF. Correlação entre a idade da menopausa e a idade da menarca em mulheres climatéricas do distrito centro-oeste de Cuiabá-MT. Reprod Clim 2001; 16:207-10.

2. Obermeyer CM. Menopause across cultures: a review of the evidence. Menopause 2000; 7:184-92.

3. van Noord PA, Dubas JS, Dorland M, Boersma H, te Velde E. Age at natural menopause in a populationbased screening cohort: the role of menarche, fecundity, and lifestyle factors. Fertil Steril 1997; 68:95-102.

4. Whelan EA, Sandler DP, McConnaughey DR, Weinberg CR. Menstrual and reproductive characteristics and age at natural menopause. Am J Epidemiol 1990; 131:625-32.

5. Dicker RC, Greenspan JR, Strauss LT, et al. Complications of abdominal and vaginal hysterectomy among women of reproductive age in the United States. The Collaborative review of Sterilization. Am J Obstet Gynecol 1982; 144:841-8.

6. Shoham Z. Should prophylatic oophorectomy be performed on post-menopausal women undergoing laparotomy or laparoscopy for non-gynaecological indications? Hum Reprod 1997; 12:201-2.

7. Oliveira VN, Valente JG, Medeiros SF. Hábitos e estilo de vida em mulheres climatéricas de baixa renda em Cuiabá. Reprod Clim 2001; 16:253-8.

8. Cassol G, Silva PB, Pinto CS, et al. Avaliação da depressão no climatério utilizando a escala de Zung. Reprod Clim 1997;12 (Supl 1):55.

9. Iannetta $O$, Rodrigues $C$, Tavares $A B$, Ferreira RA. Caracterização da estrutura familiar e dos valores pessoais num inquérito sobre a sexualidade da mulher no climatério. Reprod Clim 1997; 12 (Supl 1):49.

10. Halbe HW, Fonseca AM, Assis JS, et al. Aspectos epidemiológicos e clínicos em 1319 pacientes climatéricas. Rev Ginecol Obstet 1990; 1:182-94.

11.Faúndes A, Costa RG, Pádua KS, Perdigão AM. Associação entre prevalência de laqueadura tubária e características sócio-demográficas de mulheres e seus companheiros no Estado de São Paulo, Brasil. Cad Saúde Pública 1998; 14 (Supl 1):49-57. 
12.Instituto Brasileiro de Geografia Estatística (IBGE). Censo demográfico Brasil 1991. Rio de Janeiro: O Instituto; 2000.

13.Gentile GP, Kaufman SC, Helbig DW. Is there evidence for a post-tubal sterilization syndrome? Fertil Steril 1998; 69:179-86.

14. Souza AZ, Fonseca AM, Izzo VM, Clauzet RM, Salvatore CA. Ovarian histology and function after total abdominal hysterectomy. Obstet Gynecol 1986; 68:847-9.

15.Vessey MP, Villard-Mackintosh L, McPherson K, Coulter A, Yeates D. The epidemiology of hysterectomy: findings in a large cohort study. $\mathrm{Br}$ J Obstet Gynaecol 1992; 99:402-7.

16.Oldenhave A, Jaszmann LJ, Everaerd WT, Haspels AA. Hysterectomized women with ovarian conservation report more severe climacteric complaints than do normal climacteric women of similar age. Am J Obstet Gynecol 1993; 168:765-71.
17.Vitahmaki T, Sailahti R, Twimala R. Porque mulheres pós-menopausadas interrompem a terapia de reposição hormonal. Matúritas-Brasil 2000; 2:15-20.

18.Parazzini F, Braga C, La Vecchia C, Negri E, Acerboni S, Franceschi S. Hysterectomy, oophorectomy in premenopause, and risk of breast cancer. Obstet Gynecol 1997; 90:453-6.

19.Sightler SE, Boike GM, Estape RE, Averette HE. Ovarian cancer in women with prior hysterectomy: a 14-year experience at the University of Miami. Obstet Gynecol 1991; 78:681-4.

20.Pokras R, Hufnagel VG. Hysterectomy in the United States, 1965-84. Am J Public Health 1988; 78:852-3.

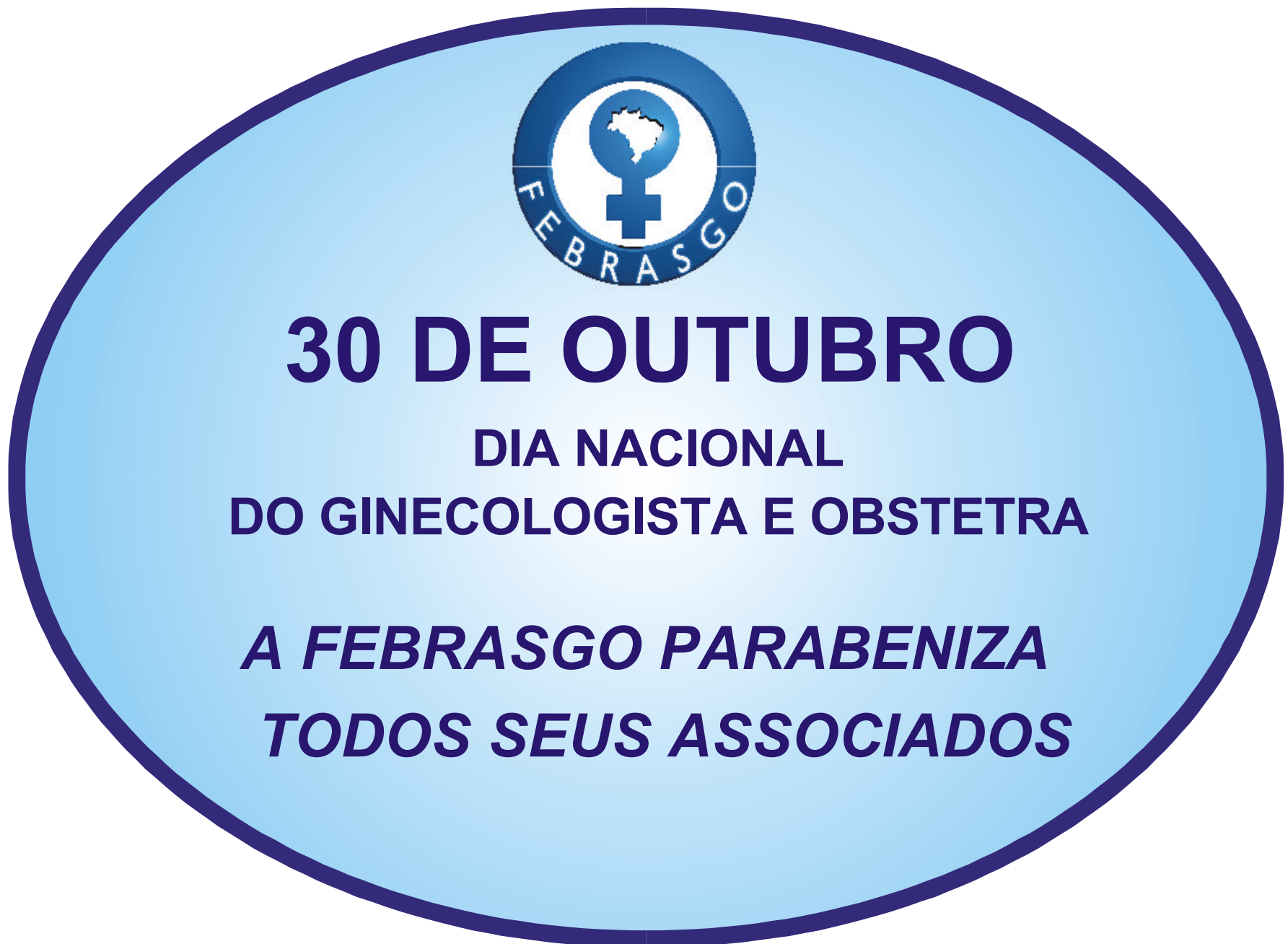

